\title{
Adaptation à la sécheresse et notion d'idéotype chez le blé dur. I. Caractères morphologiques d'enracinement
}

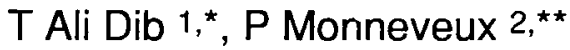 \\ 1 Université de Tichrine, faculté d'agronomie, Lattaquié, Syrie; \\ 2 ENSA-INRA, station d'amélioration des plantes, chaire de phytotechnie, 2 place Viala, 34060 Montpellier Cedex 01, France
}

(Reçu le 12 juillet 1991; accepté le 14 avril 1992)

\begin{abstract}
Résumé - Des variétés locales de blé dur bien adaptées aux conditions de l'Algérie et du Moyen-Orient, et des variétés améliorées, ont été comparées pour différents caractères morphologiques racinaires (profondeur, masse et volume des racines, répartition sur le profil); ces études ont été conduites sur sol reconstitué d'une part, et en culture aéroponique d'autre part. Les caractéristiques d'état de l'eau (teneur en eau, potentiel hydrique), en partie liées à la capacité d'extraction par les racines, ont également été mesurées au niveau des feuilles.

Les résultats obtenus permettent de distinguer plusieurs idéotypes racinaires; les relations entre les caractéristiques racinaires et les variables d'état de l'eau sont analysées, et le rôle de ces caractéristiques racinaires dans l'adaptation au milieu est discuté.
\end{abstract}

Triticum durum = blé dur / sécheresse / paramètre racinaire / culture aéroponique / idéotype

Summary - Adaptation to drought and the concept of ideotypes in durum wheat. I. Morphological traits of rooting. Durum wheat local landraces well adapted to the climatic conditions of Algeria and the Middle East and improved varieties have been compared for different morphological traits of their roots (depth, weight and volume, repartition along the soil profile); these studies have been made on reconstituted soil and in aeroponic cultures. Water status characteristics (water content, water potential) partially related to the extraction capacity of the roots have been measured in the leaves. Data obtained permit several root ideotypes to be distinguished: the relationship between root characteristics and water status parameters has been analyzed, and the role of root characteristics in adaptation to environmental conditions has been discussed.

Triticum durum = durum wheat / drought / rooting trait / aeroponic culture / ideotype

\section{INTRODUCTION}

Les possibilités d'extraction de l'eau du sol par les céréales sont, en zones sèches, très étroitement liées à la dynamique de croissance des racines (Richards et Passioura, 1981) : des relations entre certaines caractéristiques racinaires et la tolérance au déficit hydrique ont d'ailleurs été mises en évidence chez le blé dur (Benlaribi et al, 1990) et chez l'orge (Khaldoun et al, 1990).

Pourtant, malgré leur rôle essentiel dans l'alimentation minérale et hydrique et dans l'adaptation à la sécheresse, les caractères d'enracine- ment des céréales à paille ont été relativement peu étudiés, en particuler en ce qui concerne leur variabilité génétique. Les recherches portant sur les racines se heurtent de fait à de nombreux obstacles :

- difficulté d'observation et de description du système racinaire (Baldy, 1973);

- multiplicité des paramètres morphologiques racinaires (Benlaribi et al, 1990): profondeur maximale des racines, masse et volume du système racinaire, nombre de racines et finesse de l'enracinement, rapidité d'installation du système racinaire, répartition des racines sur le profil;

\footnotetext{
* Adresse actuelle: ENSA-INRA, station d'amélioration des plantes, chaire de phytotechnie, 2 place Viala, 34060 Montpellier cedex 01, France

** Correspondance et tirés à part
} 
- fluctuation importante de ces paramètres sous l'effet de divers facteurs de l'environnement, dont la contrainte hydrique (Cruiziat, 1974);

- existence de liaisons génétiques et physiologiques entre les paramètres racinaires et certains caractères agronomiques comme l'alternativité (Baldy, 1973), la hauteur du chaume (Hurd, 1974); l'indice de tallage (Masle, 1980) ou la précocité (Grignac, 1987); il est difficile, dans de telles conditions, de distinguer, dans la tolérance au déficit hydrique, le rôle des racines du rôle des caractères associés.

L'analyse des rapports entre paramètres morphologiques racinaires et comportement en conditions sèches se heurte par ailleurs à la nature même de la contrainte: la sécheresse, qui peut être définie comme une combinaison complexe de contraintes hydriques et thermiques en interaction, peut en effet prendre des formes différant considérablement d'un lieu à l'autre, ou d'une année à l'autre (Baldy, 1986). Dans de telles conditions, il apparaît illusoire de vouloir définir un idéotype de plante susceptible de s'adapter à ces différentes situations, et des paramètres morphologiques racinaires idéotypiques assurant un maintien optimal de l'absorption racinaire dans une vaste gamme d'états et de profils hydriques du sol.

La présente étude, conduite sur des variétés et populations locales d'origines très différentes (Afrique du Nord, Moyen-Orient), a pour objectifs : - de décrire les paramètres morphologiques racinaires d'adaptation à la sécheresse chez des populations locales d'Algérie et de Syrie, et chez des variétés améliorées, tolérantes à la sécheresse et à adaptation large;

- d'analyser le rôle de ces caractères dans l'adaptation de ces variétés à leur milieu, en considérant les caractéristiques particulières des zones d'origine des variétés locales étudiées. Tout en ayant des pluviométries annuelles moyennes très voisines (de l'ordre de $400 \mathrm{~mm}$ ), l'Algérie et la Syrie présentent en effet des répartitions très différentes : en Syrie, près de $60 \%$ des pluies de l'année sont des pluies hivernales et les pluies de printemps sont quasiment inexistantes (Lignon et Auriau, 1957), alors qu'en Algérie, la pluviométrie hivernale ne représente que $30 \%$ de la pluviométrie annuelle, les céréales pouvant parfois recevoir des pluies relativement abondantes au printemps (Baldy, 1986).

Une comparaison plus complète de ces génotypes, incluant l'étude de paramètres physiologiques liés à l'osmorégulation, la transpiration et la photosynthèse sera présentée ultérieurement.

\section{MATÉRIEL ET MÉTHODES}

\section{Matériel végétal}

L'étude a porté sur cinq génotypes de blé dur (Triticum durum, DESF):

- Haurani est une population locale, cultivée depuis des siècles en Syrie (Djezireh, Plaines du Nord, Hauran) et dans le Nord-Est de la Jordanie (d'Irbid à Karak). C'est un blé de type Syriacum, à épi blanc, court et très compact, à barbes courtes, à paille demipleine résistante à la verse et de hauteur moyenne (Grignac, 1965). De nombreuses lignées ont été tirées de cette population locale, en particulier Haurani 27, utilisée dans cet essai.

- Oued Zenati 368 (variété botanique leucomelan), lignée sélectionnée dans une population de blé "Bidi» de la région de Constantine (village de Oued Zenati), a été vulgarisée à partir de 1936 et occupe, depuis, la première place parmi les blés durs cultivés en Algérie. L'épi est blanc, long, assez compact; le chaume est long mais rigide et assez résistant à la verse;

- Hebda 3 (variété botanique leucomelan), est une lignée sélectionnée en 1905 par Ducellier à l'intérieur d'une population de pays; Hedba (en Arabe "Plateau"), surtout cultivée sur les hautes plaines semi-arides de l'Est algérien (pluviométrie annuelle moyenne comprise entre 400 et $500 \mathrm{~mm}$ ), est bien adaptée à ces zones souvent soumises à l'action des gels tardifs. L'épi est blanc, semi-compact.

- Belikh 2 et Sham 1 sont des variétés créées par I'ICARDA (International Center for Agricultural Research in Dry Areas, Alep, Syrie); tolérantes à la sécheresse, elles sont plus productives que Haurani (Nachit, 1987).

Les principales caractéristiques de ces 5 génotypes sont données sur le tableau I.

\section{Techniques utilisées}

Parmi les nombreuses techniques préconisées pour l'étude des racines (Baldy, 1973), 2 ont été retenues et leurs résultats comparés :

- culture en sacs plastiques sur sol reconstitué;

- culture aéroponique.

L'essai sur sol reconstitué est réalisé comme suit : les plantules des 5 génotypes, obtenues après germination sur boîte de Petri, sont repiquées dans des sacs plastiques percés à la partie inférieure pour éviter l'hypoxie (diamètre : $18 \mathrm{~cm}$; profondeur : $45 \mathrm{~cm}$ ), contenant 8 I d'un mélange de terre argilo-sableuse, de sable et de terreau (en proportion 0,5:8,5:1,0) de faible capacité de rétention ( $15 \%$ environ).

L'essai comporte au départ 8 sacs pour chaque variété, chaque sac ne recevant qu'une seule plante. Les plantules sont arrosées avec une solution nutritive jusqu'au stade 3 feuilles. À partir de ce stade, la moitié 
Tableau I. Principales caractéristiques agronomiques des 5 génotypes de blé dur étudiés (résultats d'essais réalisés en 1989-1990 sur le domaine ENSA de Lavalette, Montpellier).

\begin{tabular}{|c|c|c|c|c|c|c|c|c|}
\hline Génotype & Origine & $\begin{array}{c}\text { Levée-épiaison } \\
(j)^{\star}\end{array}$ & $\begin{array}{l}\text { Hauteur } \\
\text { (cm) }\end{array}$ & $\begin{array}{c}\text { Sensibilité } \\
\text { à la verse } \\
(0-5)\end{array}$ & $\begin{array}{c}\text { Indice } \\
\text { de tallage }\end{array}$ & $\begin{array}{l}\text { Fertilité } \\
\text { de l'épi }\end{array}$ & $\begin{array}{l}\text { Poids de } \\
1000 \text { grains } \\
\text { (g) }\end{array}$ & $\begin{array}{l}\text { Indice de } \\
\text { récolte }\end{array}$ \\
\hline Haurani 27 & $\begin{array}{l}\text { local, } \\
\text { Syrie }\end{array}$ & $\begin{array}{c}128 \\
(1203)\end{array}$ & 96 & 0,5 & 3,2 & 44 & 48 & 0,30 \\
\hline Oued Zenati 368 & $\begin{array}{l}\text { local, } \\
\text { Algérie }\end{array}$ & $\begin{array}{c}136 \\
(1330)\end{array}$ & 125 & 5,0 & 2,6 & 53 & 55 & 0,23 \\
\hline Hedba 3 & $\begin{array}{l}\text { local, } \\
\text { Algérie }\end{array}$ & $\begin{array}{c}133 \\
(1282)\end{array}$ & 110 & 1,0 & 3,0 & 49 & 57 & 0,32 \\
\hline Belikh 2 & $\begin{array}{l}\text { amélioré, } \\
\text { Icarda }\end{array}$ & $\begin{array}{c}126 \\
(1174)\end{array}$ & 90 & 0,0 & 3,7 & 45 & 51 & 0,34 \\
\hline Sham 1 & $\begin{array}{l}\text { amélioré, } \\
\text { Icarda }\end{array}$ & $\begin{array}{c}125 \\
(1159)\end{array}$ & 83 & 0,0 & 3,9 & 46 & 52 & 0,40 \\
\hline
\end{tabular}

* Les valeurs dans les parenthèses correspondent aux sommes des températures journalières moyennes (en degré-jours).

des sacs est maintenue à une humidité constante, équivalente au tiers environ de la teneur à la capacité au champ (celle-ci étant déterminée par pesée selon la méthode de Mattar, comm pers). Pour ce faire, on utilise une dose d'arrosage de $150 \mathrm{ml}$ d'eau courante par sac, apportée 2 fois par semaine. L'autre moitié ne reçoit qu'une demi-dose d'arrosage le $8^{\mathrm{e}} \mathrm{j}$.

La culture aéroponique a été réalisée selon la technique de Truong et Beunard, 1978, sur une cuve (130 $\mathrm{cm} \times 79 \mathrm{~cm} \times 98 \mathrm{~cm}$ ) couverte de plaques jointives de PVC, perforées pour recevoir les plantules; la solution nutritive, vaporisée grâce à un humidificateur type Defensor 505 placé au fond de la cuve, est une solution de Hoagland à laquelle ont été rajoutés du fer (citrate de fer: $25 \mathrm{~g} . \mathrm{I}^{-1}$ ) et de la silice (silicate de sodium: 35,3 g. ${ }^{-1}$ ). Les plantules obtenues par culture sur sable, sont repiquées au hasard, au stade 2 feuilles, à raison de 14 plantules par variété.

Les 2 expérimentations sont menées en serre (photopériode $15 \mathrm{~h}$, éclairement $75 \mathrm{~W} . \mathrm{m}^{-2}$, température $20^{\circ} \mathrm{C}$ le jour $/ 15^{\circ} \mathrm{C}$ la nuit, humidité relative $70 \%$ ).

\section{Mesures}

L'essai sur sol reconstitué est interrompu lorsque les plantes ont atteint le stade $B_{2}$ de Jonard (début montaison). Les sacs sont découpés verticalement et vidés de leur contenu; les systèmes racinaires sont rincés à plusieurs reprises.

L'essai en culture aéroponique est, quant à lui, récolté au stade fin tallage. Dans les 2 cas ont été mesurés:
- la profondeur maximale des racines (PMR);

- le nombre de racines principales de plus de $1 \mathrm{~cm}$, partant du plateau de tallage (NRP);

- le volume racinaire total (VR), évalué par immersion, selon la technique décrite par Musick et al (1965);

- la masse de substance sèche (MSSR), après séchage à l'étuve $\left(80^{\circ} \mathrm{C}, 48 \mathrm{~h}\right)$.

Par ailleurs, dans le cas de l'essai sur sol reconstitué, les volumes racinaires mesurés correspondent à 4 classes de profondeur: $A=0-10 \mathrm{~cm}, B=10-20 \mathrm{~cm}$, $C=20-30 \mathrm{~cm}$, et $\mathrm{D}>30 \mathrm{~cm}$.

Des mesures ont également été effectuées sur les parties aériennes en vue d'évaluer les rapports établis, chez les différentes variétés, entre croissance des parties aériennes et souterraines, et d'analyser les relations entre croissance racinaire et état de l'eau dans les feuilles.

Dans le premier essai, ont été mesurés :

- la masse de substance sèche (MSSPA), après séchage à l'étuve $\left(80^{\circ} \mathrm{C}, 48 \mathrm{~h}\right)$;

- la surface de la dernière feuille au stade B2, à l'aide d'un planimètre électronique Lambda LI-3000;

- la teneur en eau des feuilles;

- le potentiel hydrique foliaire; celui-ci a été mesuré à la presse à membrane, selon la méthode de Renard et Ndayishimie (1982). Les valeurs sont exprimées en $\mathrm{MPa}$.

Dans le second essai, n'ont été considérés que la masse de substance sèche aérienne et le nombre de talles. 


\section{RÉSULTATS}

\section{Essai sur sol reconstitué}

Les mesures effectuées sur les racines (tableau II) révèlent un effet variétal hautement significatif sur l'ensemble des paramètres morphologiques; l'effet du déficit hydrique appliqué est hautement significatif sur le nombre de racines principales $(-14 \%)$ et sur le volume racinaire $(-27 \%)$, et il est significatif sur la profondeur maximale des racines $(-6 \%)$, et sur la masse de substance sèche racinaire $(-17 \%)$. L'interaction traitement $x$ génotype non significative exprime le fait qu'il n'y a pas de différence entre variétés en ce qui concerne la réaction au traitement hydrique, le classement variétal étant similaire pour tous les paramètres. Dans les 2 traitements, 3 groupes peuvent être aisément distingués :

- Haurani 27 présente la profondeur maximale la plus élevée; la masse et le volume de son système racinaire sont assez importants, par contre le nombre de ses racines principales est réduit.
- Oued Zenati 368 et Hedba 3 ont, au contraire, un grand nombre de racines principales; la profondeur maximale des racines est inférieure à celle de Haurani 27. Le volume et la masse racinaire de ces deux génotypes sont très élevés;

- Belikh 2 et Sham 1 ont un système racinaire peu développé, que ce soit en masse, en volume, ou en profondeur.

L'étude de la répartition des volumes de racines par tranche de sol de $10 \mathrm{~cm}$ (fig 1) permet de mettre en évidence des différences génotypiques importantes : les variétés Oued Zenati 368 et Hedba 3 ont une part importante de leur volume racinaire total comprise dans l'horizon 0-10 $\mathrm{cm}(51 \%$ et $48 \%$ respectivement, en l'absence de déficit hydrique, contre $44 \%$ en moyenne pour les 3 autres génotypes). Chez toutes les variétés, le déficit hydrique appliqué dans notre expérimentation affecte plus la croissance volumique des couches superficielles que celle des couches profondes $(-29 \%$ en moyenne pour l'horizon $0-10 \mathrm{~cm},-22 \%$ seulement pour l'horizon supérieur à $30 \mathrm{~cm}$ ).

Tableau II. Paramètres morphologiques racinaires des 5 génotypes étudiés en culture sur sol reconstitué, sans déficit hydrique $(\mathrm{SDH})$ et avec déficit hydrique $(\mathrm{ADH}) ; P M R=$ profondeur maximale des racines; $N R P=$ nombre des racines principales; $V R=$ volume racinaire; $M S S T=$ masse de substance sèche racinaire. $H S=$ hautement significatif; $S=$ significatif; NS = non significatif. Les valeurs comportant lamême lettre ne sont pas significativement différentes au seuil de $5 \%$ (test de Newman-Keuls).

\begin{tabular}{|c|c|c|c|c|c|c|c|c|}
\hline \multirow{2}{*}{ Génotype } & \multicolumn{2}{|c|}{$P M R(\mathrm{~cm})$} & \multicolumn{2}{|c|}{$N R P$} & \multicolumn{2}{|c|}{$V R\left(\mathrm{~cm}^{3}\right)$} & \multicolumn{2}{|c|}{$\operatorname{MSSR}(g)$} \\
\hline & $S D H$ & $A D H$ & $S D H$ & $A D H$ & $S D H$ & $A D H$ & $S D H$ & $A D H$ \\
\hline Haurani 27 & $58,5^{a}$ & $55,0^{a}$ & $12,3 b c$ & $11,3^{b}$ & $0,75^{b}$ & $0,60^{b}$ & $0,12 \mathrm{ab}$ & $0,10^{a}$ \\
\hline Oued Zenati 368 & $53,5^{b}$ & $47,0^{b}$ & $14,5^{\mathrm{a}}$ & $13,0^{a}$ & $0,98^{a}$ & 0,69 a & $0,14^{a}$ & $0,11^{a}$ \\
\hline Hedba 3 & $46,0^{\mathrm{C}}$ & $41,3^{c}$ & $13,0^{b}$ & $11,0^{b}$ & $0,93^{a}$ & $0,70^{a}$ & $0,13 a$ & $0,11 \mathrm{a}$ \\
\hline Belikh 2 & $51,5^{b}$ & $51,0 a b$ & $11,5^{\mathrm{c}}$ & $9,0^{C}$ & $0,61^{c}$ & $0,40^{d}$ & $0,11^{b}$ & $0,07^{c}$ \\
\hline Sham 1 & $45,5^{c}$ & $38,8^{c}$ & $12,8 \mathrm{bc}$ & $10,0 \mathrm{bc}$ & $0,70^{b}$ & $0,50^{c}$ & $0,11^{b}$ & $0,09 \mathrm{~b}$ \\
\hline Moyenne & 50,3 & 47,3 & 12,7 & 10,9 & 0,79 & 0,58 & 0,12 & 0,10 \\
\hline Effet génotype & \multicolumn{2}{|c|}{ HS } & \multicolumn{2}{|c|}{ HS } & \multicolumn{2}{|c|}{ HS } & \multicolumn{2}{|l|}{ HS } \\
\hline Effet traitement & \multicolumn{2}{|c|}{$s$} & \multicolumn{2}{|c|}{ HS } & \multicolumn{2}{|c|}{ HS } & \multicolumn{2}{|l|}{$S$} \\
\hline $\begin{array}{l}\text { Interaction } \\
\text { traitement } \\
\text { génotype }\end{array}$ & \multicolumn{2}{|c|}{ NS } & \multicolumn{2}{|c|}{ NS } & \multicolumn{2}{|c|}{ NS } & \multicolumn{2}{|c|}{ NS } \\
\hline
\end{tabular}




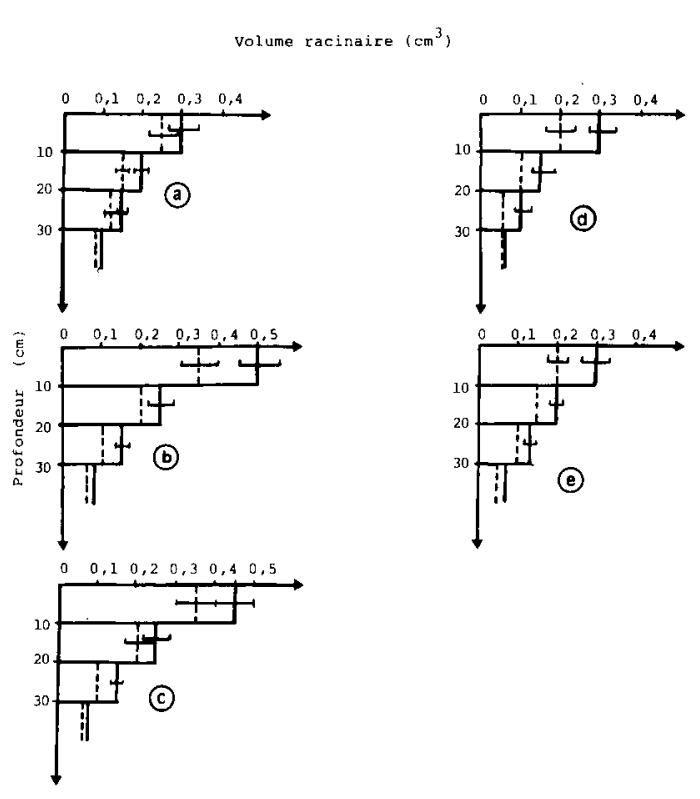

Fig 1. Répartition de volume racinaire $(e n \mathrm{~cm})$ par tranches du sol de $10 \mathrm{~cm},(-)$ sans déficit hydrique; (--..----) avec déficit hydrique. (a Haurani 27; b Oued Zenati 368; c Hedba 3; d Belikh 2; e Sham 1).

Les effets du déficit hydrique sur la croissance foliaire et sur la teneur en eau des parties aériennes ont été étudiés sur les 5 génotypes (tableau III). On peut noter :

- la surface foliaire élevée de la variété Oued Zenati 368;

- la réduction importante de la croissance foliaire, en cas de déficit hydrique, chez les 2 variétés algériennes Oued Zenati 368 et Hedba 3;

- la diminution importante de la teneur en eau des feuilles de ces 2 variétés lorsqu'elles sont soumises à la contrainte hydrique.

L'étude de l'évolution du potentiel hydrique de la feuille $\left(\psi_{F}\right)$ au cours de la phase de contrainte hydrique (fig 2) révèle par ailleurs qu'à l'issue du traitement, le potentiel hydrique est significativement (seuil $5 \%$ ) plus élevé chez les variétés du Moyen-Orient (Sham 1, Belikh 2, Haurani 27) que chez les variétés algériennes Hedba 3 et Oued Zenati 368.

\section{Essai en culture aéroponique}

Les mesures effectuées sur les racines à l'issue de l'essai en culture aéroponique (tableau IV) permettent de distinguer 3 groupes de génotypes :

- Haurani 27 : profondeur maximale des racines la plus élevée, volume racinaire significativement plus faible que chez les variétés algériennes;

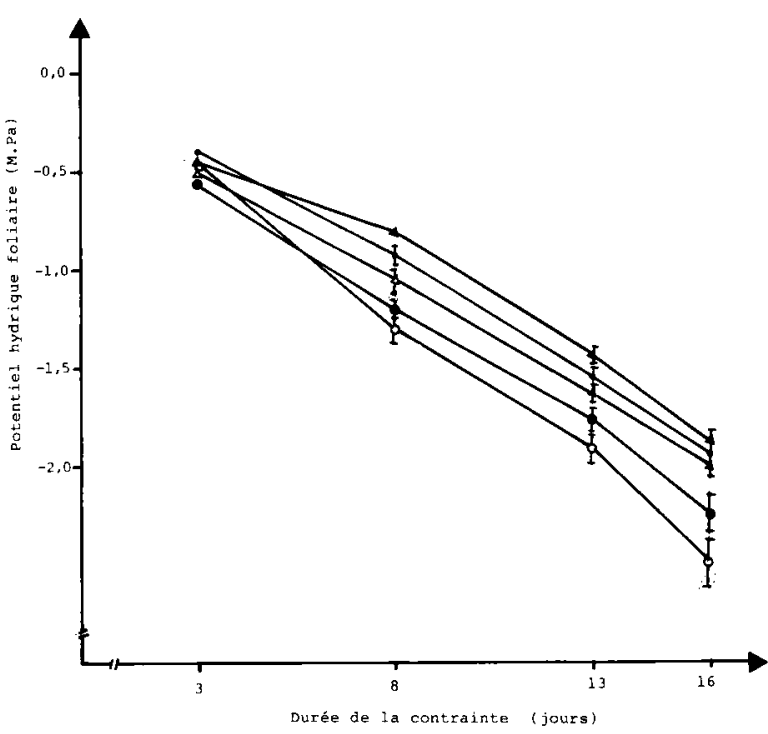

Fig 2. Évolution du potentiel hydrique foliaire au cours de la contrainte hydrique, chez les 5 génotypes étudiés. Les intervalles de confiance ne sont indiqués sur la figure que lorsque les différences entre variétés sont significatives. $\Delta$ Haurani 27; O Oued Zenati 368; - Hedba 3; • Belikh 2; A Sham 1.

- Oued Zenati 368 et Hedba 3 : enracinement profond, masse et volume racinaire élevés;

- Belikh 2 et Sham 1 : enracinement peu profond, masse et volume racinaire faibles.

En ce qui concerne les parties aériennes, on note un tallage important chez les variétés améliorées Belikh 2 et Sham 1, ainsi qu'une production de biomasse aérienne significativement plus élevée chez les variétés algériennes que chez les variétés du Moyen-Orient.

\section{DISCUSSION}

Les résultats obtenus confirment l'importante variabilité des caractéristiques racinaires à l'intérieur de l'espèce blé dur, déjà constatée par Hurd, 1974, et Benlaribi et al (1990).

Les 2 essais, réalisés selon des techniques très différentes, conduisent à des résultats très similaires; la culture aéroponique, méthode moins lourde et permettant éventuellement des observations non destructives en cours de végétation, apparaît très intéressante pour la comparaison des paramètres racinaires entre génotypes : elle a d'ailleurs déjà été utilisée pour le criblage variétal chez d'autres espèces, comme le riz pluvial (Reyniers et Jacquot, 1978) ou l'arachide (Khalfaoui, 1991).

La profondeur d'enracinement la plus grande est observée, dans les 2 essais, chez Haurani 


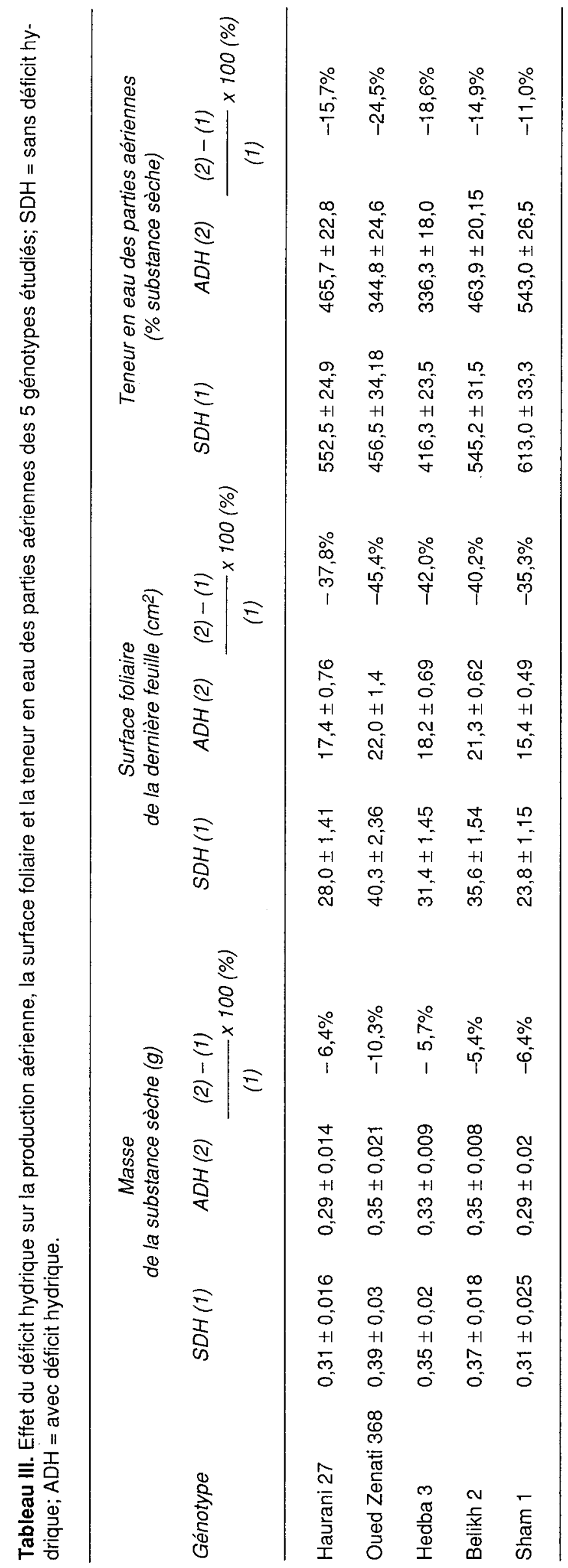


Tableau IV. Paramètres morphologiques mesurés sur les 5 génotypes en culture aéroponique; $P M R=$ profondeur maximale des racines; $N R P=$ nombre de racines principales; $V R=$ volume racinaire; $M S S R=$ masse de substance sèche racinaire; $N T=$ nombre de talles; $M S S P A$ = masse de substance sèche des parties aériennes. Les valeurs caractérisées par la même lettre ne sont pas significativement différentes entre elles au seuil de signification de $5 \%$ utilisé par le test de Newman-Keuls.

\begin{tabular}{lllllll}
\hline Génotype & PMR $(\mathrm{cm})$ & NRP & VR $\left(\mathrm{cm}^{3}\right)$ & MSSR $(g)$ & NT & MSSPA (g) \\
\hline Haurani 27 & $30,8^{\mathrm{a}}$ & $8,1^{\mathrm{a}}$ & $0,83^{\mathrm{b}}$ & $0,04^{\mathrm{ab}}$ & $2,0^{\mathrm{a}}$ & $0,10^{\mathrm{c}}$ \\
Oued Zenati 368 & $27,4^{\mathrm{ab}}$ & $9,1^{\mathrm{a}}$ & $1,06^{\mathrm{a}}$ & $0,05^{\mathrm{a}}$ & $2,07^{\mathrm{a}}$ & $0,16^{\mathrm{a}}$ \\
Hedba 3 & $26,8^{\mathrm{ab}}$ & $9,6^{\mathrm{a}}$ & $1,12^{\mathrm{a}}$ & $0,04^{\mathrm{b}}$ & $2,07^{\mathrm{a}}$ & $0,14^{\mathrm{b}}$ \\
Belikh 2 & $25,9^{\mathrm{ab}}$ & $8,6^{\mathrm{a}}$ & $0,89^{\mathrm{b}}$ & $0,03^{\mathrm{b}}$ & $2,57^{\mathrm{a}}$ & $0,13^{\mathrm{b}}$ \\
Sham 1 & $23,4^{\mathrm{b}}$ & $8,2^{\mathrm{a}}$ & $0,72^{\mathrm{b}}$ & $0,03^{\mathrm{b}}$ & $2,43^{\mathrm{a}}$ & $0,11^{\mathrm{c}}$ \\
\hline
\end{tabular}

27; même si cette profondeur n'est atteinte que par une seule des racines principales, on peut admettre, conformément aux conclusions de Callot et al (1983), que celle-ci augmente alors sa capacité d'absorption par unité de masse lorsque seuls les horizons profonds restent humectés, et se révèle ainsi capable d'assurer une contribution substantielle à l'alimentation hydrique de la plante. La profondeur d'enracinement la plus faible est, à l'opposé, observée chez la variété Sham 1.

Le nombre de racines principales apparaît plus élevé chez les variétés algériennes (Oued Zenati 368, Hedba 3) que chez les autres génotypes testés, même si la fluctuation importante de ce caractère ne permet pas toujours de dégager des différences intervariétales significatives. Le déficit hydrique affecte le nombre de racines principales chez tous les génotypes, mais les variétés algériennes semblent toutefois plus touchées que la variété Haurani 27. Les variétés Oued Zenati 368 et Hedba 3 se caractérisent également, dans les 2 essais, par une masse et un volume racinaires supérieurs à ceux des autres génotypes. L'étude, dans le cas de l'essai sur sol reconstitué, de la répartition du volume racinaire par tranche de sol de $10 \mathrm{~cm}$, permet enfin de constater que ces variétés ont un meilleur enracinement superficiel; Hurd, 1974, avait déjà noté, en comparant plusieurs variétés de blé dur, le très bon enracinement superficiel de la variété Pelissier, variété d'origine algérienne proche de Hedba 3 (Laumont, in Erroux, 1949).

L'effet de la contrainte hydrique sur le volume racinaire est particulièrement important dans le cas des variétés algériennes au système racinaire volumineux en conditions d'alimentation hydrique non limitantes; ce fait, déjà observé par Benlaribi et al (1990), conduit à formuler l'hypothèse selon laquelle l'enracinement puissant de certaines variétés pourrait leur conférer, vis-àvis de la tolérance à la sécheresse, un avantage lié autant à la contribution des racines aux pertes en eau de la plante qu'au rôle de celles-ci dans l'absorption hydrique (Cruiziat, 1974).

Le rapport d'allométrie $(R / A)$ des masses de substance sèche des parties racinaires aux masses de substance sèche des parties aériennes a été étudié dans les 2 essais (tableau V). Les variations génétiques de ce rapport, et

Tableau V. Valeurs du rapport $R / A$ de la matière sèche racinaire à la matière sèche aérienne, obtenues dans les deux essais. Les valeurs caractérisées par la même lettre ne sont pas significativement différentes entre elles au seuil de signification de $5 \%$ utilisé par le test de Newman-Keuls.

\begin{tabular}{llc}
\hline Génotype & $\begin{array}{c}\text { Essai sur } \\
\text { col reconstitué }\end{array}$ & $\begin{array}{c}\text { Essai en culture } \\
\text { aéroponique }\end{array}$ \\
\hline Haurani 27 & $0,37^{\mathrm{a}}$ & $0,40^{\mathrm{a}}$ \\
Oued Zenati 368 & $0,34^{\mathrm{bc}}$ & $0,31^{\mathrm{b}}$ \\
Hedba 3 & $0,35^{\mathrm{ab}}$ & $0,29^{\mathrm{b}}$ \\
Belikh 2 & $0,25^{\mathrm{d}}$ & $0,23^{\mathrm{c}}$ \\
Sham 1 & $0,32^{\mathrm{c}}$ & $0,27^{\mathrm{bc}}$ \\
\hline
\end{tabular}


les rapprochements qui peuvent être établis entre paramètres racinaires (volume, masse, profondeur) d'une part et paramètres de développement végétatif aérien (taille, biomasse) d'autre part, confirment l'hypothèse émise par divers auteurs (Lupton et Bingham, 1970; Cholick et al, 1977; Pepe et Welsh, 1979) selon laquelle il n'existe pas de relation génétique simple entre ces 2 catégories de paramètres. On note toutefois que les valeurs les plus faibles de ce rapport sont obtenues dans le cas des variétés Belikh 2 et Sham 1; Jaradat et Duwayri, 1981 ont également trouvé des valeurs très faibles du rapport R/A chez les variétés demi-naines Cocorit C71 ot Stork «S» (comparées dans leurs essais aux populations locales Deir Alla 2 et Haurani) : ces résultats conduisent à admettre que les gènes de nanisme affectent au moins autant le système souterrain que la partie aérienne de la plante.

La comparaison entre paramètres morphologiques racinaires et caractères agronomiques permet par ailleurs de constater :

- l'absence de relation entre l'importance de l'enracinement (volume, masse, profondeur) et l'indice de tallage (tableaux I et IV) : les variétés améliorées Belikh 2 et Sham 1, à tallage abondant, ont les systèmes racinaires les plus réduits;

- l'absence de relation simple entre profondeur d'enracinement et précocité : la variété précoce Haurani 27 a ici le système racinaire le plus profond. Même si l'on observe souvent un meilleur enracinement chez les variétés tardives (Derera et al, 1969; Grignac, 1987), il apparait donc bien que, dans l'ensemble, les paramètres morphologiques racinaires sont relativement peu dépendants génétiquement et/ou physiologiquement des principaux caractères agronomiques (taille, nombre de talles, biomasse aérienne) : cette constatation conduit à penser qu'il doit être possible de modifier génétiquement les paramètres racinaires sans entraîner de modifications corrélatives des caractères agronomiques.

L'étude de certains paramètres morphologiques de la partie aérienne (surface de la dernière feuille, masse de substance sèche des parties aériennes, potentiel hydrique foliaire, teneur en eau), et de leurs variations sous l'effet de la contrainte hydrique, montre que les variétés algériennes Oued Zenati 368 et Hedba 3 se caractérisent par :

- une surface foliaire élevée et une masse végétative importante;
- une réduction significative (seuil $5 \%$ ) de la croissance des parties aériennes, et en particulier des dernières feuilles, sous l'effet du stress hydrique (tableau III) : ce comportement vis-à-vis d'un déficit hydrique avait déjà été noté par Benlaribi et al (1990) chez les variétés algériennes Bidi 17, Hedba 2 et Gam Goum Rekham; nous avons relevé précédemment que cette réduction de croissance intéressait également, chez ces variétés, les parties souterraines;

- une réduction importante du potentiel hydrique foliaire (fig 2), alors que les variétés du MoyenOrient étudiées (Haurani 27, Belikh 2, Sham 1) maintiennent ce potentiel à un niveau plus élevé : la capacité remarquable de Haurani 27 à limiter la réduction de son potentiel hydrique en cas de contrainte a été également signalée par Seropian et Planchon (1984).

\section{CONCLUSION}

Si l'on se réfère aux différents types d'adaptation à la contrainte hydrique décrits par Turner (1986) on constate donc que les variétés du MoyenOrient étudiées ici (Haurani 27, Belikh 2 et Sham 1) présentent une double stratégie d'évitement lié à leur précocité (tableau I), et de tolérance avec maintien d'un potentiel hydrique élevé (fig 2). D'après le même auteur, un tel comportement implique un ensemble de caractéristiques morphophysiologiques : profondeur et densité racinaire élevées, réduction des surfaces évapotranspirantes, résistances stomatiques et cuticulaires élevées. Certaines de ses caractéristiques apparaissent présentes chez la variété locale Haurani 27 (enracinement profond, limitation de la surface foliaire); dans le cas des variétés améliorées Belikh 2 et Sham 1, l'introduction de gènes de nanisme réduit la masse, le volume et la profondeur racinaire, tout en contribuant à un meilleur indice de récolte (tableau I) et à une meilleure expression des potentialités de rendement en conditions favorables (Ali Dib, non publié). Une partie de ces observations (concernant en particulier la comparaison entre les variétés locales algériennes et la variété Sham 1) a d'ores et déjà été confirmée dans les conditions du champ (Bahloul, comm pers). Les variétés d'Afrique du Nord semblent par ailleurs réagir à la contrainte hydrique sévère imposée dans nos essais par un abaissement simultané du potentiel hydrique et de la teneur en eau des tissus. Une meilleure compréhension de ces 2 types de comportement implique donc une étude appro- 
fondie des paramètres morphophysiologiques liés à la transpiration, à la photosynthèse et à l'osmorégulation.

\section{RÉFÉRENCES}

Baldy C (1973) Progrès récents concernant l'étude du système racinaire du blé (Triticum $\mathrm{sp}$ ). Ann Agron 24, 241-276

Baldy C (1986) Comportement des blés dans les climats méditerranéens. Ecol Mediterr, $\mathrm{t}$ XII, fasc 3-4, 73-88

Benlaribi M, Monneveux P, Grignac P (1990) Étude des caractères d'enracinement et de leur rôle dans l'adaptation au déficit hydrique chez le blé dur (Triticum durum DESF). Agronomie 10, 305-322

Callot G, Chamayou H, Maertens C, Salsac L (1983) Mieux comprendre les interactions sol-racine. Incidence sur la nutrition minérale. INRA, Paris, $325 p$

Cholick FA, Welsh JR, Vernon-Cole C (1977) Rooting patterns of semi-dwarf and tall winter wheat cultivars under dryland field conditions. Crop Sci 17, 637-640

Cruiziat $P$ (1974) Détermination des pertes en eau subies par les différents organes d'une plante soumise au desséchement. Ann Agron 25, 539-554

Derera NF, Marshall DR, Balaam LN (1969) Genetic variability in root development in relation to drought tolerance in spring wheats. Exp Agric 5, 327-337

Erroux J (1949) Classification des blés durs cultivés en Algérie. Travaux botaniques dédiés à R Maire. Mem Soc His Nat Afr Nord, t II, Alger, $95 \mathrm{p}$

Grignac P (1965) Contribution à l'étude de Triticum durum. DESF Thèse de Doctorat, Univ Toulouse, $152 \mathrm{p}$

Grignac $P$ (1987) Tolérance au déficit hydrique et aux hautes températures de cultivars de blé. ATP écophysiologie du blé. Rapp intermédiaire campagne 1985, INRA, 279-289

Hurd EH (1974) Phenotype and drought tolerance in wheat. Agric Météorol 14, 39-55

Jaradat A, Duwayri M (1981) Effect of different moisture deficits on durum wheat seed germination and seedling growth. Cereal Res Commun 9, 55-62
Khaldoun A, Chéry J, Monneveux (1990) Étude des caractères d'enracinement et de leur rôle dans l'adaptation au déficit hydrique chez l'orge (Hordeum vulgare L). Agronomie 10, 369-379

Khalfaoui JL (1991) Approche de l'amélioration génétique de l'adaptation à la sécheresse. Cas de l'arachide au Sénégal. In: L'amélioration des plantes pour l'adaptation aux milieux arides (AUPELFUREF, ed) John Libbey Eurotext, Paris 51-63

Lignon R, Auriau $P$ (1957) Étude préliminaire sur les populations de blés cultivées dans le procheOrient. Ann Am des Plantes 1, sér B, 19-45

Lupton FGH, Bingham J (1970) Annu Rep Plant Breed Inst. Cambridge, UK, 34-37

Masle J (1980) L'élaboration du nombre d'épis chez le blé d'hiver. Influence des différentes caractéristiques de la structure du peuplement sur l'utilisation de l'azote et de la lumière. Thèse, INA-PG, Paris, 201 p

Musick GJ, Fairchild ML, Ferguson VL, Zuber MS (1965) A method of measuring root volume in corn (Zea mays L). Crop Sci 5, 601-602

Nachit M (1987) Durum wheat breeding. Cereal Improvment Program. ICARDA Annu Rep 40-57

Pepe JF, Welsh HR (1979) Soil water depletion patterns under dryland field conditions of closely related height lines of winter wheat. Crop Sci 19, 677-680

Renard C, Ndayishimie V (1982) Étude des relations hydriques chez Coffea arabica L. I. Comparaison de la presse à membrane et de la chambre à pression pour la mesure du potentiel hydrique foliaire (psi). Café Caco Thé 26, 27-30

Reyniers FN, Jacquot M (1978) Démarche pour l'obtention de la résistance variétale à la sécheresse : cas du riz pluvial. Agron Trop 33, 314-317

Richards RA, Passioura JB (1981) Seminal root morphology and water use of wheat. II. Genetic variation. Crop Sci 21, 253-255

Seropian C, Planchon C (1984) Physiological responses of six bread wheat and durum wheat genotypes to water stress. Euphytica 33, 3, 757-767

Truong B, Beunard P (1978) Étude de la croissance racinaire de six variétés de riz pluvial en culture aéroponique. Agron Trop 33, 213-236

Turner NC (1986) Adaptation to water deficits: a changing perspective. Aust J Plant Physiol 13, 175-190 